\title{
Glucose-enhanced performance by aged rats on a test of conditional discrimination learning
}

\author{
GORDON WINOCUR \\ Rotman Research Institute, Baycrest Centre for Geriatric Care, North York, Ontario, Canada \\ and Trent University, Peterborough, Ontario, Canada
}

\begin{abstract}
Groups of normal old rats and young adult rats were administered a test of conditional discrimination learning (CDL) with variable intervals between stimulus presentation and response selection. Previous work with this task showed that CDL acquisition depends on frontal-lobe involvement, whereas the abil ity to recall conditional stimuli at relatively long intervals requires an intact hippocampus. Old rats were slower to learn the CDL habit and their impairment increased with the length of the stimulus-response interval. Peripheral injections of glucose $(100 \mathrm{mg} / \mathrm{kg})$ improved performance of old rats at delays of 5 and $15 \mathrm{sec}$, but had no effect in the 0-sec delay condition. Young rats did not respond to glucose treatment. The enhancing effects of glucose in the old group were found to correlate positively with measures of glucose metabolism. The results provide evidence that glucose-induced attenuation of cognitive loss in old rats is especially pronounced for specific memory function under hippocampal control.
\end{abstract}

There is growing evidence that loss of learning and memory associated with normal aging can be attenuated by glucose treatment (see reviews by Gold, 1991, 1992). Gold and his colleagues reported that aged rats injected with glucose performed significantly better than saline-treated controls on tests of inhibitory avoidance conditioning (Gold, Vogt, \& Hall, 1986) and spontaneous alternation (Stone, Rudd, \& Gold, 1992). In studies of human populations, normal old people obtained higher scores on memory tests after drinking a glucose-sweetened beverage than they did after drinking the same beverage sweetened with saccharin (Manning, Hall, \& Gold, 1990; Manning, Parsons, \& Gold, 1992). There is also evidence that the beneficial effects of glucose extend to the memory disorders of patients with Alzheimer's disease. In recent work, patients in different stages of the disease displayed significant improvement on a variety of recall and recognition tests after glucose ingestion (Craft, Zallen, \& Baker, 1992; Manning, Ragozzino, \& Gold, 1993).

These effects have been attributed to a breakdown in the process whereby epinephrine, a hormone released peripherally during learning, produces increased amounts of circulating glucose that crosses the blood-brain barrier and influences brain function. As glucose tolerance diminishes with age, there is a decline in the amount of glucose that is taken up by the brain, and a corresponding decline in function (Gold, 1991, 1992). Glucose treatment appears to facilitate glucose uptake, resulting in improved brain function.

This research was supported by a grant from the Natural Sciences and Engineering Research Council of Canada. I am grateful to Morris Moscovitch for helpful comments on an earlier version of this paper, and to John Zomer for his able technical assistance. Correspondence should be directed to G. Winocur, Rotman Research Institute, Baycrest Centre for Geriatric Care, 3560 Bathurst Street, North York, ON M6A 2EI, Canada (e-mail: winocur@psych.utoronto.ca).
The hippocampus (HPC) and related structures, known to be involved in the mediation of learning and memory, have been identified as critical sites of glucose action. Decreased cholinergic activity within the hippocampal system is associated with profound memory loss, and one possibility is that glucose injections compensate for such deficits by facilitating the synthesis of acetylcholine. In support of this view, it has been noted that glucose, administered to humans with various types of memory problems, is most effective in improving performance on tests that are sensitive to medial temporal lobe/hippocampal dysfunction (Manning et al., 1990; Manning et al., 1992; Manning et al., 1993). In mice, spontaneous alternation deficits induced by the cholinergic antagonist scopolamine were reversed by peripheral (Stone et al., 1992) or central (Parsons \& Gold, 1992) administration of glucose.

In recent years, a primary focus of our research program has been the development of behavioral paradigms that are capable of assessing differential effects of damage to various brain structures on learning and memory. One test that has proven to be especially useful for this purpose is a conditional discrimination learning (CDL) task in which rats must learn to associate different responses with different stimuli. This is a variation of Petrides's conditional learning paradigm that has proven to be highly sensitive to effects of damage to the prefrontal cortex (PFC) in monkeys (Petrides, 1982). In the present test, which is conducted in an operant chamber, the rat is presented with one of several visual stimuli and is required to select the correct lever to obtain a food reward.

In a previous study, the CDL task was administered to groups of rats with lesions to the PFC or HPC (Winocur, 1991). The PFC group was severely impaired in learning the conditional discrimination when the presentation of the stimulus was followed immediately by the appearance of the levers. Its performance was not affected, relative to 
controls, when the interval between the interval and the opportunity to respond was increased. In contrast, rats with HPC lesions learned the conditional discrimination rule as well as the control group when the stimulus-response interval was very brief, but their performance deteriorated dramatically at longer intervals.

Normal old rats were also administered the CDL task and were found to be impaired relative to young adults (Winocur, 1992). Interestingly, separate measures of performance revealed signs of both HPC and PFC dysfunction. That is, like rats with $\mathrm{PFC}$ damage, old rats had difficulty learning the CDL rule and, like rats with HPC lesions, their performance declined disproportionately at longer delays. As would be expected, the age-related deficits, while similar in kind, were not as severe as those seen in young adult rats with discrete brain lesions.

The CDL task is well suited to an investigation of the attenuating effects of glucose on age-related cognitive impairment. As indicated above, there is evidence that glucose administration improves performance of aged animals and humans on tests of episodic and declarative memory that involve the HPC system. A few studies investigated the effects of glucose on frontal-lobe function, with generally negative results (Manning et al., 1990; Craft, Murphy, \& Wemstrom, 1994). These studies were conducted exclusively with human subjects and did not include tests of conditional learning. By providing separate measures of hippocampally mediated memory function and a form of learning for which the PFC is essential, the CDL task allows an assessment of whether glucose effects are beneficial to performance in general or whether they are restricted to specific learning and memory processes.

\section{METHOD}

\section{Subjects}

Thirty male Long-Evans rats, obtained from the Trent University Breeding Centre, served as subjects for the experiment. At the beginning of the experiment, the old rats $(N=16)$ were 23 months old and the young adult rats $(N=14)$ were 6 months old. As young adults, the old rats had participated in experiments involving spatial memory or discrimination learning. To equate prior experience, the young control rats were administered the same spatial memory task several weeks before the beginning of this study. None of the subjects had prior experience with an operant conditioning task.

The old rats lived in group cages, housing $4-6$ rats, until one month before the experiment, when they were transferred to individual wire-mesh cages. The young rats had lived in individual cages since weaning. Water was available at all times, but food was provided in accordance with experimental conditions. The rats were examined regularly by a veterinarian to ensure that they were healthy and abie to participate in the study.

\footnotetext{
Apparatus

All testing was conducted in five identical operant chambers, each outfitted with two retractable levers, a centrally located display panel, and a food-well. The display panel consisted of a bank of two rows of two miniature lamps, each $1 \mathrm{~cm}$ in diameter and spaced $3 \mathrm{~cm}$ apart. The food-well was situated centrally, $5 \mathrm{~cm}$ below the lights and between the levers. A pellet dispenser delivered $45 \mathrm{mg}$ Noyes food pellets to the food-well. Three walls and the ceiling of each box were made of Plexiglas. The fourth wall, containing the levers, etc. was made of metal, and the floor consisted of steel rods spaced $1.5 \mathrm{~cm}$ apart.
}

\section{Procedure}

Three weeks before the beginning of training, the rats were handled regularly and gradually reduced to about $80 \%$ of normal body weight. Throughout the experiment, they were maintained at constant weight by being fed $20-25 \mathrm{~g}$ of lab chow each day. The experiment began with a three-stage training program.

Stage 1 . The rats received daily sessions in which they were trained to press both levers according to a continuous reinforcement (CRF) schedule. In each session, a rat could obtain a maximum of 160 food pellets, distributed equally across left and right leverpresses. When one lever was pressed 80 times, that lever remained in the chamber but subsequent presses of that lever no longer delivered pellets. Each session in Stage 1 ended when 160 pellets were delivered, or after a 30-min time limit. The criterion for completing Stage 1 was three consecutive sessions in which the maximum number of pellets was obtained.

Stage 2. The day after criterion was reached on Stage 1, the rats advanced to Stage 2 , in which they were trained to become familiar with levers retracting after reinforcement. Each daily session began with both levers present. Pressing either lever produced a food pellet, followed by the retraction of both levers for $10 \mathrm{sec}$. The levers then reappeared and remained in the chamber until a response was performed. The cycle was then repeated. Each lever could be pressed 80 times for reinforcement. When 80 pellets had been obtained with one lever, that lever was retracted and the session continued with only the other lever operative. This procedure served to discourage the buildup of position preferences. The criterion for completing Stage 2 was three consecutive 30 -min sessions in which 160 reinforced pellets were obtained.

Stage 3. The day after criterion was reached on Stage 2, Stage 3 was instituted to train the rats to respond differentially to the presence and absence of lights on a given trial. Daily sessions consisted of 100 light and 100 no-light trials, with the order of presentation randomly determined. A light trial began with illumination of all four lights and the simultaneous appearance of both levers. When the rat pressed the lever, a pellet was dispensed, the lights turned off, and the levers retracted. A $10-\mathrm{sec}$ interval followed each light trial. In the light trials, the rats were permitted a maximum of 50 responses to each lever. When that number was reached, the lever was retracted and did not reappear in the session. In the no-light trials, both levers appeared for $10 \mathrm{sec}$ or until the rat pressed one of the levers. No pellets were provided in the no-light trials. If the rat did not press a lever during the $10 \mathrm{sec}$, both levers retracted for $10 \mathrm{sec}$. If the rat did press a lever in a no-light trial, a 30-sec intertrial interval followed.

A session was terminated when 200 trials (100 light and 100 no light) were completed. The criterion for completion of Stage 3 was three consecutive sessions in which the ratio of leverpresses in the light trials to leverpresses in the no-light trials was $2: 1$ or better.

CDL acquisition. The CDL task was introduced the day after criterion was reached on Stage 3. Each session began with the illumination of the top light, the bottom light, or both lights on either side of the light panel. The lights served as discriminative stimuli, and their onset coincided with the appearance of both levers. The levers remained in the chamber until a response was performed. A stimulus on the left side of the panel was the signal for pressing the left lever, while a stimulus on the right side was the signal for a rightlever response. A correct response produced a food pellet, followed by the stimulus offset, retraction of both levers, and a 10 -sec intertrial interval. An incorrect response led to stimulus offset, the withdrawal of the levers, no reinforcement, and a 30 -sec intertrial interval. Each session consisted of 80 trials in which the left lever was correct and 80 trials in which the right lever was correct, the order being randomly determined. One 160 -trial session was administered each day for 40 consecutive days.

CDL with delay. The day after the last CDL-acquisition session, a delay condition was instituted. The procedure was the same for this condition, except that the number of trials was reduced to 150 per 
session and, on two-thirds of the trials, a delay was introduced between the offset of the discriminative stimulus and the appearance of the levers. For 50 trials, the delay was $5 \mathrm{sec}$ and for another 50 trials, the delay was $15 \mathrm{sec}$; the remaining 50 trials ( $0-\mathrm{sec}$ delay) were identical to those of the CDL-acquisition condition. The order of delay trials was determined by one of three sequences that rotated daily. The sequences were random except for the requirement that each session began with a 0 -sec-delay trial. The rats received 10 daily sessions in the delay condition.

CDL and treatment. The day after CDL-with-delay testing, the rats received the first of 10 daily sessions in which they were injected peripherally with $100 \mathrm{mg} / \mathrm{kg}$ of glucose or an equal volume of saline between 20 and $30 \mathrm{~min}$ before testing. This dosage was selected on the basis of previous work that demonstrated its effectiveness in attenuating age-related memory deficits in other paradigms (Gold, 1991). On the first day of this condition, the subjects were tested following saline treatment, and then glucose and saline treatments were alternated on successive days. In other respects, testing was identical to the CDL-with-delay condition.

Blood-glucose measurement. A few days after the completion of the experiment, blood samples were taken on two separate occasions separated by $48 \mathrm{~h}$. Blood-glucose levels were measured with a glucometer (Glucometer 3; Ames Canada). Baseline glucose levels were established on each day by pricking the tail with a scalpel, drawing a drop of blood, and applying the blood to a strip of Glucofilm. About $30 \mathrm{~min}$ later, the rat was injected with saline or glucose $(100 \mathrm{mg} / \mathrm{kg})$ in counterbalanced order. Blood samples were taken again at 10-, 30-, and 60-min intervals, and glucose levels were measured in the usual way.

\section{RESULTS}

\section{Age Differences in CDL Performance}

There were no differences between the groups as they advanced through the various stages of the CDL training program, which required about three weeks to complete. In Stage 3 of training, just before CDL-acquisition trials were initiated, young and old groups took an average of 7 and 8 days, respectively, to reach the criterion of responding in the light-trials at twice the rate that they did in the no-light-trials.

The CDL-acquisition data for both groups are presented in Figure 1. The data are expressed in terms of percentage correct responses over the 40 sessions, averaged over eight blocks of five sessions. As the figure shows, the old group was much slower in learning the CDL habit and, by the end of training, had stabilized at a performance level that was considerably below that of the young group. An analysis of variance (ANOVA) applied to these data revealed significant effects of age $[F(1,28)=8.11, p<.01]$ and block $[F(7,197)=18.61, p<.001]$, and a significant age $\times$ block interaction $[F(7,197)=6.38, p<.025]$.

Figure 2A shows the groups' performance on the CDL task when delays were introduced between stimulus presentation and the appearance of the levers. The data points at each delay represent the scores for the old and young groups averaged across 5 days of testing. As can be seen in Figure $2 \mathrm{~A}$, varying the interval had little effect on the young group's performance. In contrast, the old group's accuracy rate declined dramatically at longer delays, and was no better than chance at the 15 -sec delay. These observations were confirmed by an ANOVA that yielded a significant group $\times$ delay interaction $[F(2,56)=5.31, p<.025]$. In addition, multiple comparisons with the Tukey test showed that, within the old group, there were significant differences in performance between all delays $(p s<.05)$. By comparison, performance differences between the three delays for the young group were statistically nonsignificant.

To assess the effects of delay on CDL performance, independent of differences in learning ability, a regression analysis was performed in which age and 0 -sec delay were independent variables. The difference between groups at

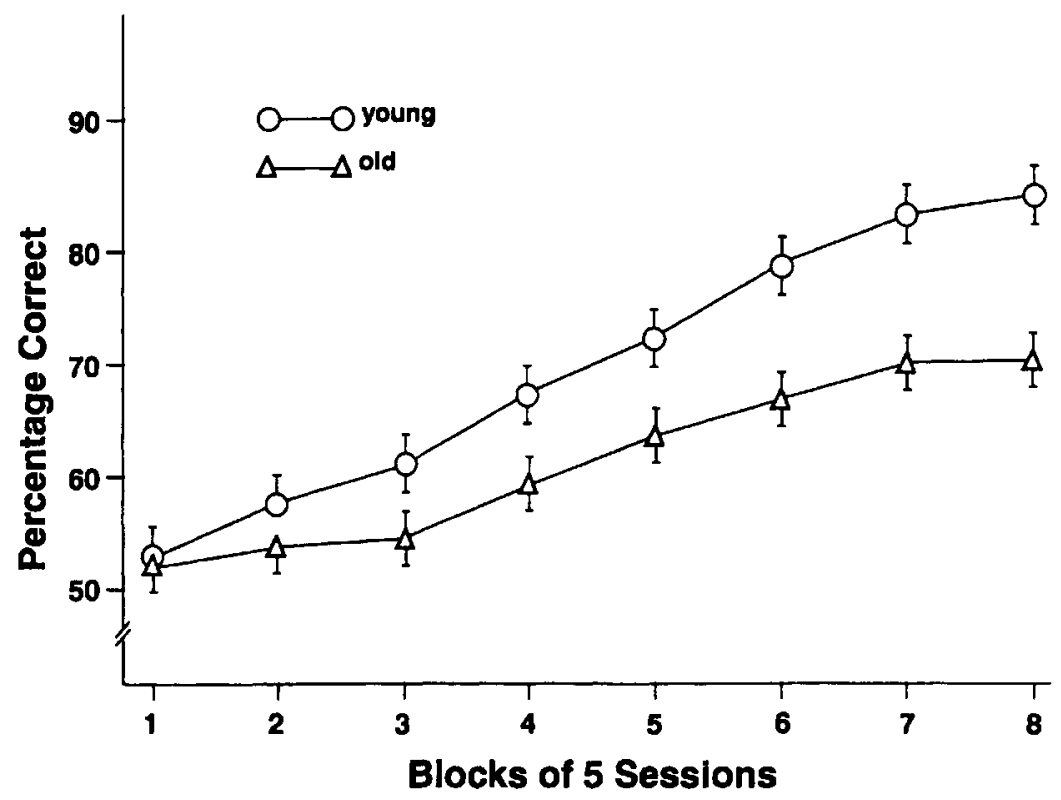

Figure 1. Performance of old and young groups during CDL acquisition. Data are averaged over eight blocks of five sessions. Values shown are group means $\pm S E M$. 


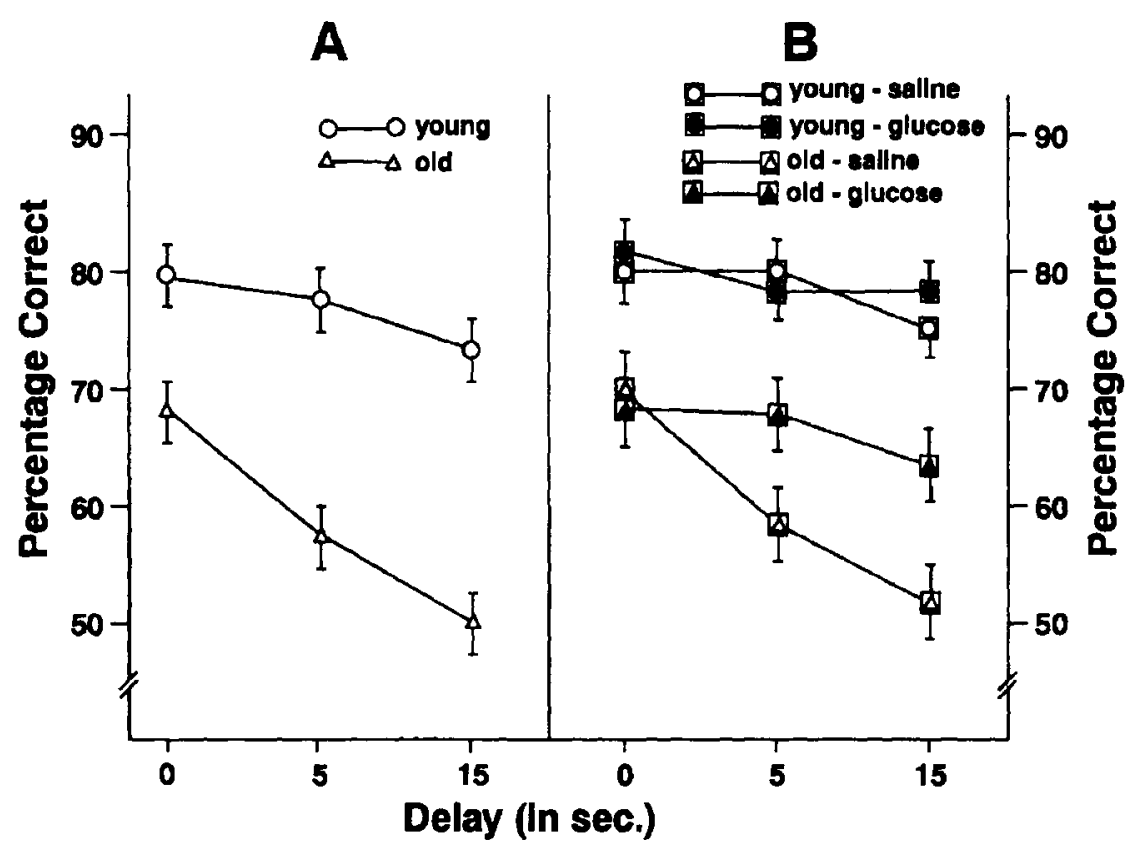

Figure 2. Performance of old and young groups in the CDL-with-delay (A) and CDL-andtreatment $(B)$ conditions. The scores represent mean values $\pm S E M$ over the total number of sessions in each condition.

0 -sec delay was used to predict differences between groups at 5- and 15-sec delays. In this way, the variance due to learning was eliminated. The differences between age groups were highly significant at both 5 -sec delay $[F(2,27)=5.84$, $p<.01]$ and $15-\sec$ delay $[F(2,27)=12.43, p<.001]$.

The effects of glucose and saline injection on CDL performance at the various delays are displayed in Figure 2B. The results indicate that the old rats selectively benefited from glucose treatment, but that the benefits occurred only at the longer delay intervals. The differential effects of glucose were confirmed by a significant age $\times$ treatment $\times$ delay interaction $[F(2,143)=4.09, p<.025]$. Main effects of age $[F(1,28)=6.37, p<.025]$, treatment $[F(1,143)=4.15, p<.05]$, and delay $[F(3,143)=2.73$, $p<.05]$ were also statistically significant.

Separate ANOVAs revealed significant group $\times$ treatment interactions at the $5-\sec [F(1,28)=4.45, p<.05]$ and $15-\sec [F(1,28)=8.02, p<.01]$ delays. At both delays, the old rats performed significantly better following glucose injection than they did following saline injection. Glucose treatment had no effect on the young group's performance at either delay. At the 0 -sec delay, the performance difference between old and young groups was statistically significant $[F(1,28)=5.89, p<.025]$, but there was no effect of treatment.

\section{Blood-Glucose Levels and Performance}

A comparison of baseline blood-glucose levels sampled at the end of testing indicated significantly lower levels in the young rats $[F(1,28)=4.33, p<.05]$. However, baseline glucose levels were not found to be related to acquisition of the CDL habit. Comparisons between glucose levels and response accuracy during each five-session block consistently yielded nonsignificant correlations in both age groups (all $p s>.05$ ). Similarly, comparisons between glucose levels and performance during CDL-withdelay testing produced no significant correlations in either group at any of the three delays.

Blood-glucose measures were taken also at 15-min, 30$\mathrm{min}$, and 60 -min intervals after injections of saline or glucose $(100 \mathrm{mg} / \mathrm{kg}$ ). (See Figure 3.) Saline injections caused blood glucose to rise at comparable rates in young and old groups. Glucose levels seemed to peak between 15 and $30 \mathrm{~min}$, and returned to baseline by $60 \mathrm{~min}$. An ANOVA applied to these data confirmed that glucose levels were higher in the old group $[F(1,28)=4.36, p<.05]$, but the group $\times$ delay interaction was not significant $[F(2,56)=$ $2.01, p>.05]$.

A different pattern of recovery was observed after glucose injection. In the young group, blood glucose rose initially and, after peaking at around $30 \mathrm{~min}$, declined to slightly above baseline levels at $60 \mathrm{~min}$. By comparison, in the old group, blood glucose returned to baseline at a much slower rate, so that $60 \mathrm{~min}$ postinjection, glucose levels were still substantially higher in the old group than in the young group. These observations were confirmed by a significant age $\times$ delay interaction $[F(2,56)=5.41$, $p<.01]$, in addition to the main effect of age $[F(1,28)=$ $10.33, p<.005]$.

To assess the relationship between glucose regulation and performance on the CDL task, an index of glucose utilization was constructed for each rat by calculating the difference between the mean of the three glucose measurements following saline injection and the mean of the three 


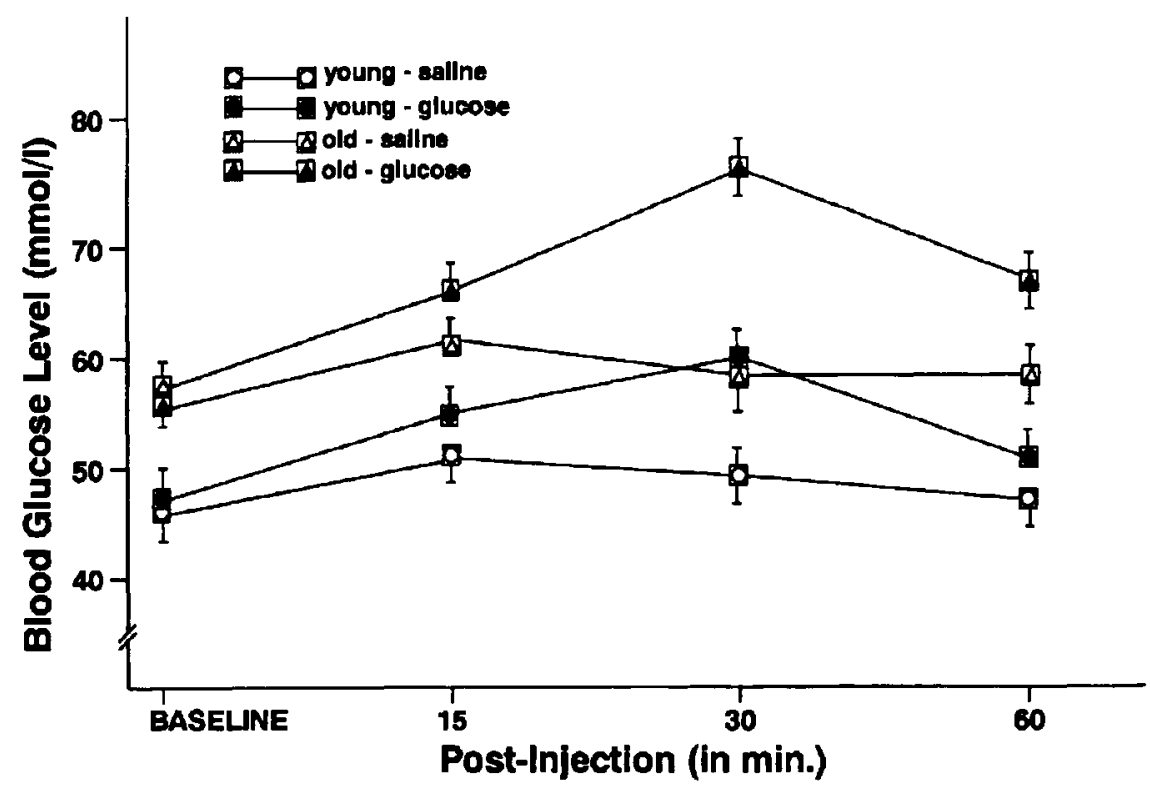

Figure 3. Blood-glucose levels (in mmol/L) in old and young groups at baseline and at 15 , 30 , and $60 \mathrm{~min}$ after injection with glucose $(100 \mathrm{mg} / \mathrm{kg})$ or saline. Values shown are group means $\pm S E M$.

glucose measurements following glucose injection (Craft et al., 1992). The glucose index for each rat was then compared against its average performance scores on the glucose treatment days. Pearson-product coefficients, shown in Table 1, revealed a significant correlation in the old group at the 15 -sec delay $(r=.56, p<.05)$, and a strong trend toward significance at the 5 -sec delay $(r=.44, p<.10)$. The correlation between the old group's glucose utilization index and performance at 0 -sec delay was not significant $(r=.33, p<.20)$. Comparisons involving the glucose index and performance in the young group were statistically nonsignificant at all three delays.

\section{DISCUSSION}

The results of this experiment confirm previous reports that peripheral injections of glucose can reduce normal age differences between young and old rats on tests of learning and memory (Gold et al., 1986; Stone et al., 1992; Stone, Wenk, Olton, \& Gold, 1990). Whereas the earlier work involved tests of inhibitory avoidance and spontaneous alternation, the present research utilized a foodmotivated test of conditional learning. The CDL task used

Table 1

Correlations Between Glucose Index and CDL Performance for Each Delay (in Seconds) Averaged Over the Five Glucose-Treatment Sessions

\begin{tabular}{cccc}
\hline & \multicolumn{3}{c}{ Delay } \\
\cline { 2 - 3 } Subjects & 0 & 5 & 15 \\
\hline Old & .33 & $.44 \dagger$ & $.56^{*}$ \\
Young & .21 & .16 & .28 \\
\hline
\end{tabular}

${ }^{*} p<.05 .{ }^{+} p<.10$. in this study was a multidimensional task in which animals were required to learn associations between different stimuli and responses. When delays were introduced between the stimuli and the opportunity to respond there was the additional requirement to remember specific stimuli as part of the process of response selection.

Studies with brain-damaged animals, that have used the CDL and similar conditional learning tasks, have provided evidence that these processes are distinct and controlled by dissociable brain mechanisms (Winocur, 1991, 1992b). The PFC appears crucial for learning basic associations in a CDL paradigm (see also Petrides, 1982), but damage to this structure does not affect performance when stimulusresponse intervals are lengthened. In contrast, the HPC is essential for retaining specific information at relatively long delays, although it does not appear to be involved in the acquisition of the CDL rule.

Normal old rats were impaired in learning the CDL task and their performance declined further when episodic memory was challenged by forcing the animal to retain specific conditional stimuli before being allowed to respond (see also Winocur, 1992). These results point to an age-related deterioration of function in PFC and HPC brain regions, and are consistent with other evidence that these structures are especially vulnerable to the effects of aging ( $\mathrm{Al}-$ bert \& Stafford, 1986; Boone, Miller, \& Lisser, 1993).

A major finding of the present study was that glucose administration selectively improved CDL performance in old rats at the 5- and 15-sec delays, but had no effect in the 0 -sec-delay condition. It is noteworthy that the facilitatory effects of glucose at the longer delays were sufficient to eliminate age differences in performance that could be attributed to failure to recall trial-specific conditional stim- 
uli. As can be seen in Figure 2B, old rats treated with glucose performed at the same level at all delay intervals. Although the old group continued to perform worse than the young group on glucose days, the difference between groups was the same at all delays and was probably related to differences in CDL acquisition.

The results are consistent with other evidence that glucose administration enhances performance on tests of episodic memory that is under hippocampal control, and supports the view that glucose action is mediated by cholinergic mechanisms (Craft et al., 1994; Gold, 1992; Manning et al., 1990). The fact that glucose failed to affect CDL performance at 0 -sec delay, a measure of procedural learning that involves PFC function, is consistent with reports based on aged human subjects (Craft et al., 1994; Manning et al., 1990), and offers strong evidence of the selectivity of glucose effects.

While there is compelling evidence that glucose acts on hippocampally related memory processes and that this effect is dissociable from other aspects of learning and memory, it may be premature to conclude that glucose effects are restricted exclusively to such processes. There has been only limited investigation of effects of glucose on tasks that are reliably associated with frontal-lobe function. Further, it should be noted that the glucose dosage selected for the present study was based on evidence of its effectiveness in reducing memory deficits associated with hippocampal function. It is conceivable that frontal-lobe functions respond to glucose treatment but that such effects are governed by different dose-response curves. It is also possible that frontal functions other than those assessed here are affected by glucose administration. At least one study showed that young adult humans performed better on various frontal-lobe tests of attention after drinking a glucose beverage than after a placebo (Benton, Owens, \& Parker, 1994). These and other issues related to the generality of glucose effects are currently under investigation in our laboratory.

The present research appears to be the first demonstration that glucose can improve memory performance in food-deprived old rats tested on an appetitive task. Glucoseenhanced performance has been reported in fasting old people (Craft et al., 1994; Manning et al., 1990), but the only comparable studies in the animal literature were conducted in young adult rats (Messier \& White, 1987) or mice (Means \& Fernandez, 1992). Since blood-glucose levels are relatively low as a result of food deprivation in such studies, one might argue that glucose treatment was acting on performance-related measures. For example, glucose-induced reduction in hunger may have helped subjects attend to essential features of the task. A second possibility that applies to the animal studies is that glucose treatment had a positive effect on subjects' affective state, which intensified the reward properties of food and provided additional incentive (Messier \& Destrade, 1988). A strong argument against these interpretations of the present results is provided by the fact that glucose improved performance at the 5 - and 15 -sec delays but had no effect on 0 -sec-delay trials, where the animal was not required to remember the conditional stimuli. A more likely interpretation is that glucose effects were mediated through brain mechanisms controlling memory-related functions.

In contrast to some reports (e.g., Means \& Fernandez, 1988; Messier \& White, 1987), there was no evidence that glucose had comparable benefits for young rats. However, the design of the present study may have masked such effects. At the completion of CDL training, young rats were performing at or near maximal levels, and further improvement would have been difficult to demonstrate. In general, the evidence with respect to the behavioral effects of glucose on young adults is inconsistent. Variations in dosage and task-related factors may account for some of the differences but, clearly, the question requires further study.

Finally, the analysis of blood-glucose levels provided some insight into possible relationships between glucose control and age-related memory loss. In line with observations of food-deprived human subjects (Craft et al., 1994; Craft et al.,1992), baseline glucose levels were significantly higher in old rats than they were in young rats. This finding suggests an age-related impairment in glucose regulation, which was also indicated by the pattern of change in blood glucose following glucose injection. Bloodglucose levels rose faster and reached a higher peak in the old rats in comparison with the young adults. The rate of recovery was also slower in old rats and, indeed, $60 \mathrm{~min}$ postinjection, blood-glucose levels were still abnormally high.

Although variations in baseline blood-glucose measures did not correlate with CDL performance, elevated glucose levels in old rats clearly coexisted with a lower level of performance. An examination of blood-glucose changes and CDL behavior following glucose treatment provided stronger evidence of a relationship between glucose regulation and cognitive performance. In this regard, the important finding was that the beneficial effects of glucose were greatest in old rats that showed the lowest peak increases in blood glucose and the fastest recovery to baseline levels. Other investigators have reported similar results. Stone et al. (1990) found an inverse correlation between increases in blood glucose following glucose injection and performance by old rats on a test of avoidance conditioning. Craft and her colleagues (Craft et al., 1994; Craft et al., 1992) found that prolonged elevation of bloodglucose levels following glucose ingestion by normal old people was associated with reduced enhancement of memory function.

Thus, the present results are consistent with existing evidence that memory loss in old age is related to poor glucose control and decreased transfer of glucose from blood to brain. By this view, abnormally high levels of circulating glucose would be needed to increase glucose uptake and support normal brain function. Interestingly, Stone et al. (1992) found that glucose treatment attenuated scopolamine-induced reduction in HPC uptake of glucose and also resulted in a faster rate of uptake in old mice than in young mice. The latter finding, which was accompanied by a corresponding improvement in spontaneous alternation behavior, suggests a sensitivity to high levels of blood glucose that facilitates glucose transport to specific brain 
regions. This sensitivity may be an important component of the mechanism whereby glucose enhances effects in individuals experiencing reduced glucose tolerance.

\section{REFERENCES}

Albert, M. S., \& STAFFord, J. L. (1986). CT scan and neuropsychological relationship in aging and dementia. In G. Goldstein \& R. E. Taske (Eds.), Advances in clinical neuropsychology (Vol. 3, pp. 3153). New York: Plenum.

Benton, D., OWens, D. S., \& Parker, P. Y. (1994). Blood glucose influences memory and attention in young adults. Neuropsychologia, 32, 595-607.

Boone, K. B., Miller, P. L., \& Lesser, I. M. (1993). Frontal lobe cognitive functions in aging: Methodologic considerations. Dementia, 4 , 232-236.

Craft, S., Murphy, C., \& Wemstrom, J. (1994). Glucose effect on complex memory and nonmemory tasks: The influence of age, sex, and glucoregulatory response. Psychobiology, 22, 95-105.

Craft, S., Zallen, G., \& Baker, L. D. (1992). Glucose and memory in mild senile dementia of the Alzheimer type. Journal of Clinical \& Experimental Neuropsychology, 14, 253-267.

GoLD, P. E. (1991). An integrated memory system: From blood to brain. In R. C. A. Frederickson, J. L. McGaugh, \& D. L. Felton (Eds.), Peripheral signaling of the brain: Role in neural-immune interactions. learning and memory (pp. 391-419). Toronto: Hogrefe \& Huber.

GoLD, P. E. (1992). Modulation of memory processing: Enhancement of memory in rodents and humans. In L. R. Squire \& N. Butters (Eds.), Neuropsychology of memory (2nd ed., pp. 402-414). New York: Guilford.

Gold, P. E., VoGT, J., \& Hall, J. L. (1986). Glucose effects on memory: Behavioral and pharmacological characteristics. Behavioral \& Neural Biology, 46, 145-155.

Manning, C. A., Hall, J. L., \& Gold, P. E. (1990). Memory facilitation by glucose in aged humans. Psychological Science, 1, 307-311.

Manning, C. A., Parsons, M. W., \& Gold, P. E. (1992). Anterograde and retrograde enhancement of 24-h memory by glucose in elderly humans. Behavioral \& Neural Biology, 58, 125-130.
Manning, C. A., Ragozzino, M. E., \& Gold, P. E. (1993). Glucose enhancement of memory in patients with probable senile dementia of the Alzheimer's type. Neurobiology of Aging, 14, 523-528.

Means, L. W., \& Fernandez, T. J. (1992). Daily glucose injections facilitate performance of a win-stay water-escape working memory task in mice. Behavioral Neuroscience, 106, 345-350.

Messier, C., \& Destrade, C. (1988). Improvement of memory for an operant response by post-training glucose in mice. Behavioural Brain Research, 31, 185-191.

Messier, C., \& White, N. M. (1987). Memory improvement by glucose, fructose, and two glucose analogs: A possible effect on peripheral glucose transport. Behavioral \& Neural Biology, 48, 104-127.

Parsons, M. W., \& Gold, P. E. (1992). Scopolamine-induced deficits in spontaneous alternation performance: Attenuation with lateral ventrical injections of glucose. Behavioral \& Neural Biology, 57, 9092.

Petrides, M. (1982). Motor conditional associative-learning after selective prefrontal lesions in the monkey. Behavioural Brain Research, $5,407-413$.

Stone, W. S., Rudd, R. J., \& Gold, P. E. (1992). Glucose attenuation of scopolamine- and age-induced deficits in spontaneous alternation behavior and regional brain $\left[{ }^{3} \mathrm{H}\right] 2$-deoxyglucose uptake in mice. Psychobiology, 20, 270-279.

Stone, W. S., Wenk, G. L., Olton, D. S., \& Gold, P. E. (1990). Poor blood glucose regulation predicts sleep and memory deficits in normal aged rats. Journals of Gerontology: Biological Sciences, 45. B169-B173.

WinOCUR, G. (1991). Functional dissociation of the hippocampus and prefrontal cortex in learning and memory. Psychobiology, 19, 11 20.

WINOCUR, G. (1992). Conditional learning in aged rats: Evidence of hippocampal and prefrontal cortex impairment. Neurobiology of Aging, 13, 131-135.

(Manuscript received February 9, 1995; revision accepted for publication April 17, 1995.) 